\title{
The importance of parameter resampling for soil moisture data assimilation into hydrologic models using the particle filter
}

\author{
D. A. Plaza ${ }^{1}$, R. De Keyser ${ }^{2}$, G. J. M. De Lannoy ${ }^{1}$, L. Giustarini ${ }^{3}$, P. Matgen ${ }^{3}$, and V. R. N. Pauwels ${ }^{1}$ \\ ${ }^{1}$ Laboratory of Hydrology and Water Management, Ghent University, Ghent, Belgium \\ ${ }^{2}$ Department of Electrical energy, Systems and Automation, Ghent University, Ghent, Belgium \\ ${ }^{3}$ Centre de Recherche Public - Gabriel Lippmann, Département Environnement et Agrobiotechnologies, \\ Belvaux, Luxembourg \\ Correspondence to: D. A. Plaza (douglasantonio.plazaguingla@ugent.be)
}

Received: 1 June 2011 - Published in Hydrol. Earth Syst. Sci. Discuss.: 21 June 2011

Revised: 5 January 2012 - Accepted: 26 January 2012 - Published: 8 February 2012

\begin{abstract}
The Sequential Importance Sampling with Resampling (SISR) particle filter and the SISR with parameter resampling particle filter (SISR-PR) are evaluated for their performance in soil moisture assimilation and the consequent effect on baseflow generation. With respect to the resulting soil moisture time series, both filters perform appropriately. However, the SISR filter has a negative effect on the baseflow due to inconsistency between the parameter values and the states after the assimilation. In order to overcome this inconsistency, parameter resampling is applied along with the SISR filter, to obtain consistent parameter values with the analyzed soil moisture state. Extreme parameter replication, which could lead to a particle collapse, is avoided by the perturbation of the parameters with white noise. Both the modeled soil moisture and baseflow are improved if the complementary parameter resampling is applied. The SISR filter with parameter resampling offers an efficient way to deal with biased observations. The robustness of the methodology is evaluated for 3 model parameter sets and 3 assimilation frequencies.

Overall, the results in this paper indicate that the particle filter is a promising tool for hydrologic modeling purposes, but that an additional parameter resampling may be necessary to consistently update all state variables and fluxes within the model.
\end{abstract}

\section{Introduction}

It is widely recognized that hydrologic models are useful tools for a number of purposes, ranging from flood forecasting (Andersson, 1992) to numerical weather prediction and climate studies (Zhang et al., 2008). Due to uncertainties in the meteorological forcings and model parameters, and errors or oversimplifications in the model physics, these models are always prone to a certain level of uncertainty. One way to reduce the predictive uncertainty of hydrologic models is to regularly update these models using externally obtained data sets, which is commonly referred to as Data Assimilation (DA). The improvement of hydrologic model results through the assimilation of soil moisture data has been the subject of numerous studies (Entekhabi et al., 1994; Walker et al., 2002; Pauwels et al., 2002; De Lannoy et al., 2007a). The underlying idea of data assimilation is to calculate a weighted average between the observations and the model results. The simplest way to perform this is to simply replace the model results by the observations, which is defined as direct insertion (Heathman et al., 2003). More advanced assimilation methods include nudging of the model results to the observations (Houser et al., 1998; Pauwels et al., 2001; Paniconi et al., 2002) and optimal interpolation (Seuffert et al., 2004). These techniques are in fact simplifications of the Kalman filter (Kalman, 1960), in which the model error is calculated explicitly throughout the simulation.

Originally developed for linear systems, and later extended for nonlinear systems, a great deal of attention has 
been paid to this assimilation method for hydrologic data assimilation. The extended Kalman filter, in which the forecast error covariance is calculated through a linearization of the model, and the ensemble Kalman filter, in which this model error covariance is calculated using the spread of an ensemble of model realizations, have been intercompared by Reichle et al. (2002). At this point, it can be argued that the ensemble Kalman filter is the most frequently used assimilation method in hydrology. A variation to this method is the ensemble Kalman smoother (Dunne and Entekhabi, 2005), in which observations that are distributed in time are used to update the model state variables. This method is comparable to variational assimilation (Caparrini et al., 2004), in which observations within a predefined window are used to estimate the initial state variables. One problem with the frequently used ensemble Kalman filter is the underlying assumption of Gaussianity of both the forecast and observation error structure. As it is evident that this assumption is not realistic for hydrologic systems, assimilation methods have been developed that relax this assumption.

One method that is receiving increasing attention in hydrology is the particle filter, which has been developed to function for any kind of model error (Liu and Chen, 1998). This method has been used to assimilate discharge records into relatively simple rainfall-runoff models (Weerts and El Serafy, 2006; Moradkhani et al., 2005b) and to assimilate water stage records into hydraulic models (Matgen et al., 2010; Giustarini et al., 2011). Recently, this method is also used for the assimilation of soil moisture data, for the estimation of model parameters (Montzka et al., 2011), and the estimation of root-zone soil moisture conditions (Nagarajan et al., 2010).

According to Moradkhani et al. (2005b), Nagarajan et al. (2010), and Montzka et al. (2011), it is clear that the trend towards the application of particle filters is not limited to only the state estimation problem, but it can also be used for the identification of model parameter values, by exploiting the advantage of the flexible structure of the particle filter algorithms. In this study, state and parameter estimation are performed within the framework of the particle filter, aiming at an improvement of the model performance in terms of both soil moisture and discharge, through the assimilation of soil moisture data. Moreover, instead of estimating all the model parameters, we propose a methodology where a limited model parameter set is used. Dual or joint estimation has been widely studied using either the Kalman filter (Moradkhani et al., 2005a; Franssen and Kinzelbach, 2008; Wang et al., 2009) or recently, the particle filter (Moradkhani et al., 2005b; Nagarajan et al., 2010; Montzka et al., 2011). The approach presented here differs from previous state-parameter estimation studies in the objective. More specifically, the particle filter with parameter resampling is applied aiming at an improvement of the modeled discharge as a result of soil moisture assimilation, and the parameter values are not estimated explicitly.
The organization of the paper is as follows: first, the study site and the description of the model are presented. The description of the experiment is presented. Then, the data assimilation methodologies are explained, after which the results from the study are explained. Finally, the conclusions from this study are summarized.

\section{Materials and methods}

\subsection{Site description}

The area (Fig. 1) to be studied is located in the Grand Duchy of Luxembourg and includes the drainage area expanded from the head of the Alzette River basin, $4 \mathrm{~km}$ south of the French-Luxembourg border, to the stream gauge located in Pfaffenthal (Luxembourg City).

The discharge area covers a surface of $356 \mathrm{~km}^{2}$ and consists of about $50 \%$ cultivated land, $22 \%$ urban centers and $28 \%$ woodland. The topography of the floodplain is characterized by a natural sandstone bottleneck which is located near Luxembourg city. The valley located upstream of the bottleneck is up to $2.5 \mathrm{~km}$ wide, while in the Luxembourg sandstone the valley is only $75 \mathrm{~m}$ wide. The geological substratum is dominated by marls on the left bank and by limestone and sandstones deposits on the right bank. Sand and gravel, as well as marls and clay alternate in the alluvial deposits covering the stratum. A gauging station, operated since 1996, is located around the village of Livange providing accumulated precipitation amounts with a sampled frequency of $15 \mathrm{~min}$. The meteorological station at Findel Airport is operated in the vicinity of the catchment.

\subsection{Model description}

The Community Land Model (CLM2.0) is the hydrologic model used in this study. CLM2.0 simulates land surface processes by calculating water and heat fluxes for each grid cell separately, without any interaction between cells. Each grid cell can be subdivided into several patches, containing one single land cover type such us urban, vegetated, wetlands, glacier and lake. The vegetated fraction is further subdivided into patches of plant functional types, which maintain their own prognostic variables (i.e., a vegetated land cover with 4 patches representing 4 different plant functional types). In this study, CLM2.0 was adapted in order to be able to use the individual patches as ensemble members according to De Lannoy et al. (2006a).

The meteorological forcings required by the model are the air temperature, wind speed, specific humidity, incoming solar radiation, and precipitation. The meteorological forcings were assumed to be spatially uniform over the complete study area. Vertical layers in CLM2.0 embody one vegetation layer, up to ten soil layers and up to 5 snow layers. In this application, soil layers depths were set to 10, 20, 30, 40, $50,60,70,80,90,100 \mathrm{~cm}$. CLM2.0 computes the surface 
Table 1. Optimal parameter sets: NwRb and NwRs were converted into tuneable parameters (De Lannoy, 2006b), $k$ is the soil layer index. NwRb and NwRs are not considered in the parameter resampling step.

\begin{tabular}{|c|c|c|c|c|}
\hline Description & set 1 & set 2 & set 3 & set 4 \\
\hline Fraction of model area with high water table (wtfact[fraction]) & 0.280 & 0.704 & 0.742 & 0.7174 \\
\hline Water table depth scale parameter $\left(\mathbf{f z}\left[\mathbf{m}^{-1}\right]\right)$ & 49.173 & 3.423 & 3.475 & 3.523 \\
\hline Saturated soil hydraulic conductivity $\left(\mathbf{k d}\left[\mathbf{m m ~ s}^{-1}\right]\right)$ & 0.827 & 0.095 & 0.099 & 0.098 \\
\hline Base flow parameter for saturated fraction of watershed $\left(\mathbf{l d}\left[\mathbf{m m ~ s} \mathbf{~}^{-1}\right]\right)$ & 0.0071 & 0.0034 & 0.0027 & 0.0038 \\
\hline First bottom layer contributing to the calculation of base flow (NwRb [-]) & 5 & 5 & 6 & 5 \\
\hline Last top layer contributing to the calculation of the surface runoff (NwRs $[-])$ & 3 & 4 & 4 & 4 \\
\hline Clapp and Hornberger constant $\left(\mathbf{b s w}_{\mathbf{k}}[-]\right)$ & 5.487 & 4.659 & 4.623 & 5.919 \\
\hline Volumetric soil water at saturation $\left(\right.$ watsat $\left._{\mathbf{k}}[-]\right)$ & 0.638 & 0.597 & 0.600 & 0.617 \\
\hline Hydraulic conductivity at saturation (hksat $\left.{ }_{\mathbf{k}}\left[\mathbf{m m ~ s}^{-1}\right]\right)$ & 0.047 & 0.011 & 0.010 & 0.024 \\
\hline Minimum soil suction $\left(\right.$ sucsat $\left._{\mathbf{k}}[\mathbf{m m}]\right)$ & 284.76 & 557.17 & 606 & 497.16 \\
\hline
\end{tabular}

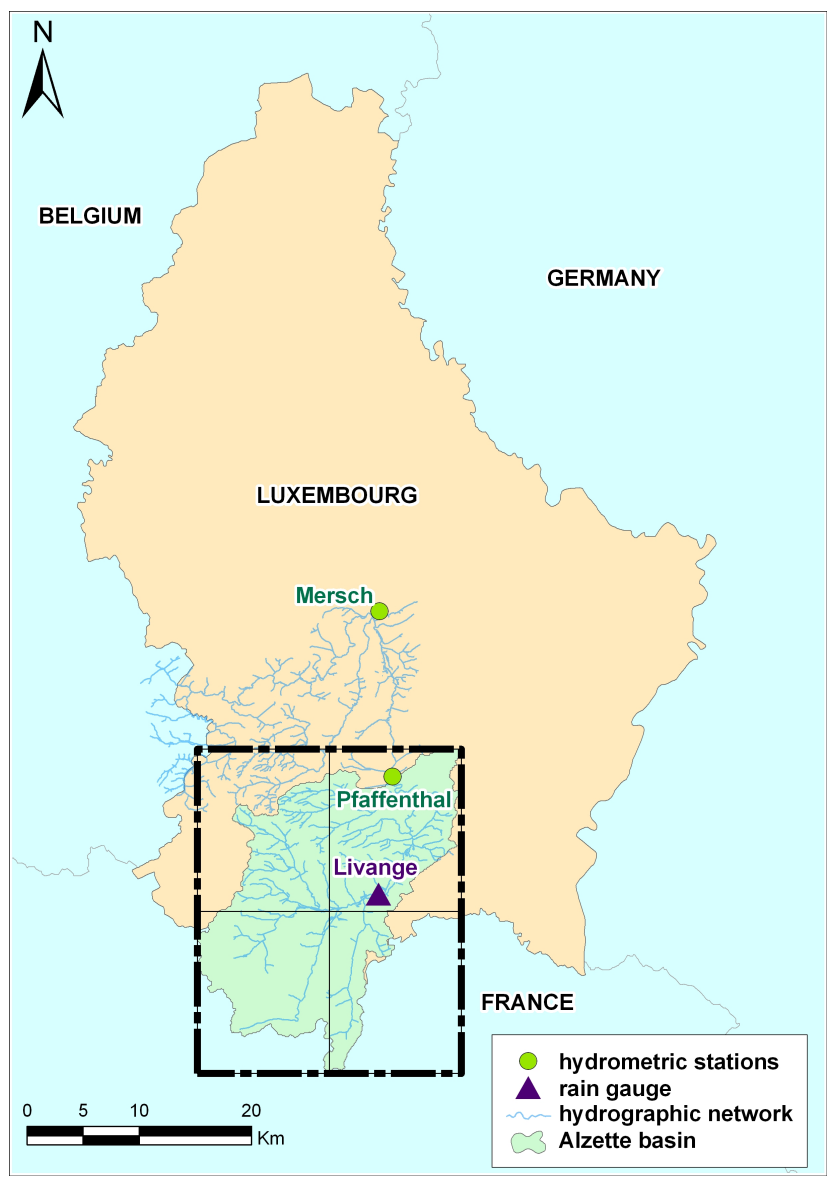

Fig. 1. The study area: the discharge area in the Alzette river basin is indicated by the green patch.

runoff and the baseflow for every grid cell. The discharge is routed to the basin outlet using the linear unit hydrograph approach of Troch et al. (1994).

In CLM2.0, each grid cell contains around 30 model parameters related to the different physical processes repre- sented by the model such as the canopy water balance, the soil water balance, and the energy balance. From these $30 \mathrm{pa}-$ rameters, 10 parameters are related to the soil water balance. The reduced parameter set allows for the application of automatic calibration algorithms, such as the Shuffled complex evolution approach (Duan et al., 1993) which was used in this study. Table 1 presents the description of the selected parameters and three corresponding sets of optimal parameter values (set 1 , set 2 , set 3 ) which yield a similar good model performance. Due to the size of the parameter space and the complexity of the model, the system is prone to behave the equifinality phenomena. The optimal values were identified by minimizing the Root Mean Square Error (RMSE) between observed and simulated discharge during the year 2006. The three parameter sets will be used to validate the data assimilation methodology.

The model is applied using a constant hourly time step and the study area is represented by 4 grid cells at a $10 \mathrm{~km}$ $\mathrm{x} 10 \mathrm{~km}$ resolution which is consistent with the resolution of large scale models. For the sake of clarity in the presentation of the algorithm performances, results corresponding to the cell located in the lower left quadrant in Fig. 1 are presented.

\subsection{Experimental setup}

A synthetic soil moisture data assimilation study is performed to assess the performance of the filters. Soil moisture assimilation has received a lot of attention during the last decades, but insight in the impact of soil moisture assimilation on dependent variables, for instance discharge, has been limited (Pauwels et al., 2002; De Lannoy et al., 2007b; Brocca et al., 2010).

For each model grid cell, synthetic volumetric soil moisture observations, corresponding to the top $10 \mathrm{~cm}$ soil layer, are generated with the CLM2.0. The generation of the observation consists of the perturbation of the model parameters presented in Table 1 (set 4) and the perturbation of the forcings. Parameters and forcings were perturbed by white 

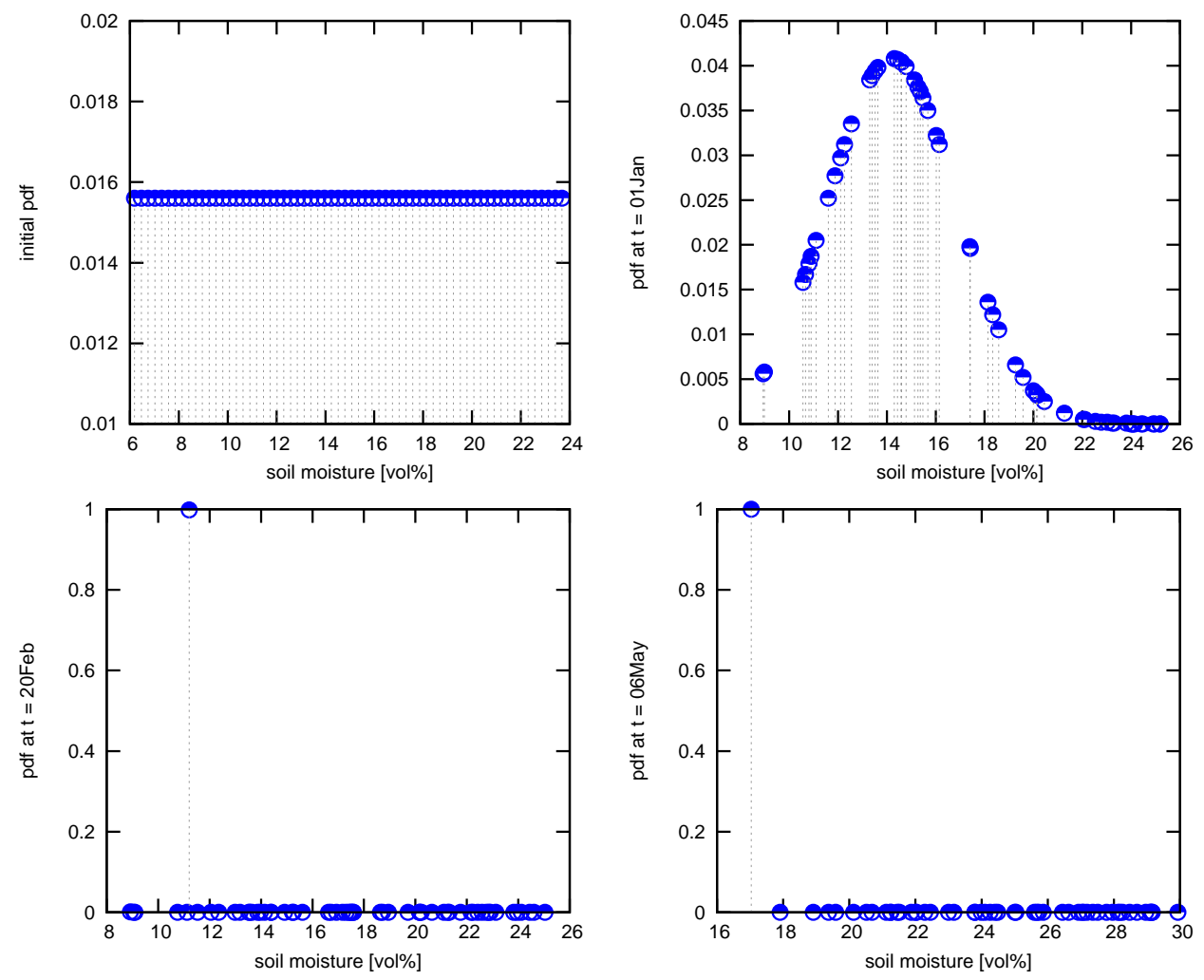

Fig. 2. Monitoring the importance weights: axis $\mathrm{x}$ represents the particles location (volumetric soil moisture [vol \%]) and axis y the importance weights values at four different daily model time steps 0, 1 (1 Jan), 51 (20 Feb), 126 (6 May).

Gaussian noise with zero mean and the standard deviation set to $1 \%$ of the parameter value and $1 \%$ of the maximum forcing value. Furthermore, it is worth mentioning the following two facts: first, the small level of noise used in the perturbation of the parameters and forcings can limit the representation of a real-case model error and second, the use of a different parameter set (set 4) in the generation of the synthetic observations introduces bias in the observations themselves. Therefore, the synthetic experiment is focused more on the study of the performances of the filters in the removal of bias and may not represent most of the real-world situations. However, the way how the experiment is carried out allows to demonstrate the applicability of the particle filter in this study-case.

The forecast uncertainty is introduced through the generation of soil moisture random samples, which is referred to ensemble generation. The meteorological forcings and the model parameters were disturbed with an additive zero mean white Gaussian noise in order to obtain the soil moisture ensemble (De Lannoy et al., 2006a). The standard deviation of this random number for the parameters was set to a predefined fraction of the parameter value. In order to check for the correctness of the ensemble, two different ensemble verification measures were used (De Lannoy et al., 2006a). The ensemble spread $\left(\mathrm{ensp}_{t}\right)$, the ensemble mean square er- ror $\left(\mathrm{mse}_{t}\right)$, and the ensemble skill $\left(\mathrm{ensk}_{t}\right)$ have to be computed first and at each time step $t$ :

$$
\begin{aligned}
\operatorname{ensp}_{t} & =\frac{1}{N} \sum_{i=1}^{N}\left(\hat{z}_{t, i}-\overline{\hat{z}}_{t}\right)^{2} \\
\operatorname{mse}_{t} & =\frac{1}{N} \sum_{i=1}^{N}\left(\hat{z}_{t, i}-z_{t}\right)^{2} \\
\operatorname{ensk}_{t} & =\left(\overline{\hat{z}}_{t}-z_{t}\right)^{2}
\end{aligned}
$$

where $\hat{z}_{t}$ is the variable to be estimated and $z_{t}$ is the corresponding observation of the estimated variable at time step $t$. In order to have a large enough ensemble spread, on average the ensemble mean differs from the observation by a value that is equal to the time average of the ensemble spread. Therefore, the following expression should be true:

$\frac{<\text { ensk }>}{<\text { ensp }>} \approx 1$

where $<$. $>$ indicates an average over the simulation period. Furthermore, if the truth is statistically indistinguishable from a member of the ensemble, the following expression should be true:

$$
\frac{<\sqrt{\mathrm{ensk}}>}{<\sqrt{\mathrm{mse}}>} \approx \sqrt{\frac{N+1}{2 N}}
$$



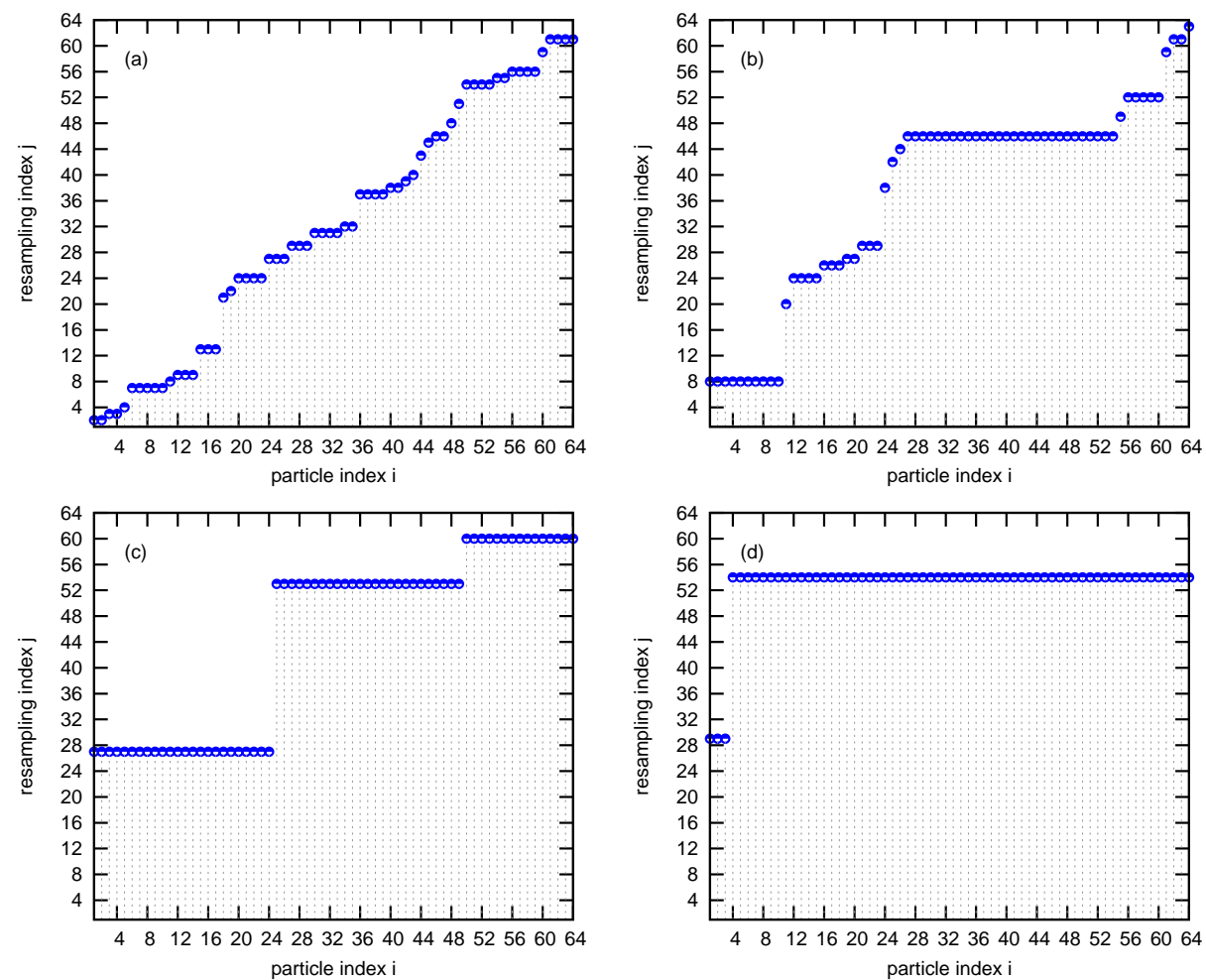

Fig. 3. Extreme particle replication example with $N=64$ : (a) and (b) present a proper resampling performance whereas (c) and (d) show the extreme replication problem. The sample set collapses to particles located at 27th, 53th and 60th positions in (c) and almost all the set collapses to the value of the particle at the 54 th position in (d).

For the selection of the ensemble/particle size, the assimilation algorithms were evaluated using three different ensemble sizes: 64, 128 and 256. Results shown that the improvement obtained when increasing the size is not very significant while the increase in the computational time demand is very significant. Therefore, an ensemble size of 64 is used in this study.

The standard deviation of the perturbation noises corresponds to $10 \%$ of the nominal values for the model parameters, and $1 \%$ for the meteorological forcings. These fractions have been calibrated in order to balance the different sensitivities of model parameters and meteorological forcings in the generation of an adequate ensemble. Figure 4 shows the soil moisture ensemble and the corresponding baseflow ensemble, the ratio $<$ ensk $>/<$ ensp $>$ is equal to 1.09 which approximates 1 and the ratio $<\sqrt{\mathrm{ensk}}>/<\sqrt{\mathrm{mse}}>$ is equal to 0.72 which approximates the value of $\sqrt{1 / 2}$ with the simulation period corresponding to year 2007 .

A robustness test of the assimilation algorithms will be performed by considering the impact of the data assimilation frequency and of different optimal parameter values for the model integration. Discussion on the filter performances for these scenarios will be extended in the results section.

\subsection{Particle filters}

In nonlinear estimation, the dynamic system in discrete time is described by the state evolution equation given by:

$\boldsymbol{x}_{t}=\boldsymbol{f}_{t}\left(\boldsymbol{x}_{t-1}, \boldsymbol{u}_{t-1}, \boldsymbol{v}_{t-1}\right)$

where $t$ is the discrete time index, $\boldsymbol{x}$ is the state vector, $\boldsymbol{f}_{t}($. is the nonlinear function, $\boldsymbol{u}$ is the input vector and $\boldsymbol{v}$ is the process noise. In this study, the state vector consists of 22 variables for each vertical profile, i.e., canopy water storage, vegetation temperature and soil temperature and moisture at 10 levels, the observed state corresponds to the soil moisture at the top layer. CLM2.0 represents the nonlinear function $\boldsymbol{f}_{t}($.$) and \boldsymbol{u}_{t}$ is the vector of meteorological forcings.

The state estimation is accomplished when the information from the measurement is assimilated into the model. The relationship between the measurements and the system states (the observation model) is given by:

$\boldsymbol{y}_{t}=\mathbf{H}_{t} \boldsymbol{x}_{t}+\boldsymbol{n}_{t}$

Equation (5) represents the observation model, where $\boldsymbol{y}$ is a vector which contains the measurements, $\mathbf{H}_{t}$ is a diagonal matrix containing values of 0 and 1 and $\boldsymbol{n}_{t}$ is the noise affecting the observations. In this study the observation model 

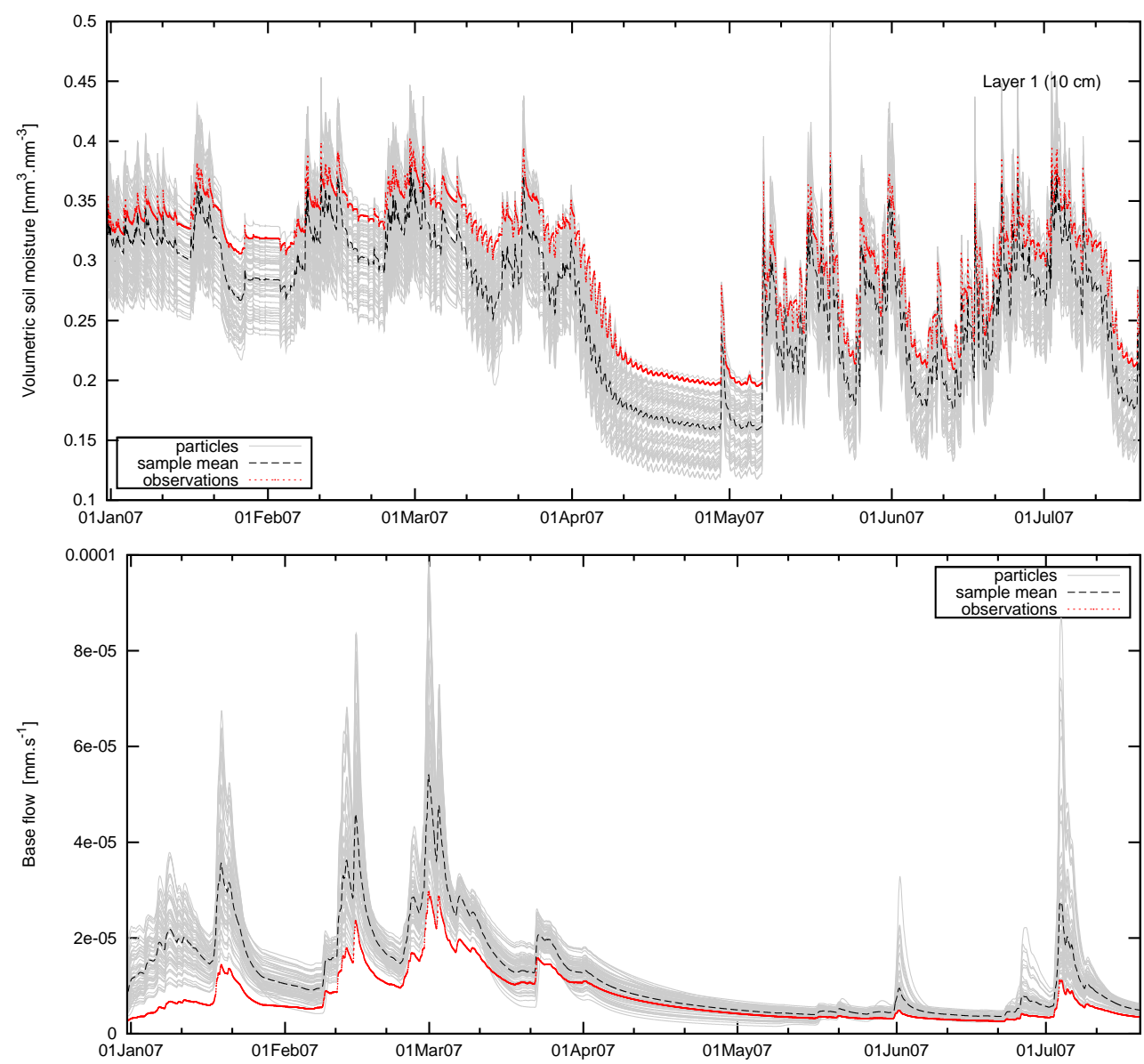

Fig. 4. Soil moisture and baseflow ensembles: The upper plot corresponds to the generation of the volumetric soil moisture ensemble with the ensemble members in gray line, the ensemble mean in black line and the synthetic soil moisture observations in red dotted line. The lower plot corresponds to the baseflow ensemble.

is linear, because the assimilated soil moisture observations will correspond directly to the soil moisture state variables.

In recursive Bayesian filtering the solution to the estimation problem consists of two steps: the prediction and correction steps. These steps are formulated as follows:

$$
\begin{aligned}
p\left(\boldsymbol{x}_{t} \mid \boldsymbol{y}_{1: t-1}\right) & =\int p\left(\boldsymbol{x}_{t} \mid \boldsymbol{x}_{t-1}\right) p\left(\boldsymbol{x}_{t-1} \mid \boldsymbol{y}_{1: t-1}\right) d \boldsymbol{x}_{t-1} \\
p\left(\boldsymbol{x}_{t} \mid \boldsymbol{y}_{1: t}\right) & =\frac{p\left(\boldsymbol{y}_{t} \mid \boldsymbol{x}_{t}\right) p\left(\boldsymbol{x}_{t} \mid \boldsymbol{y}_{1: t-1}\right)}{\int p\left(\boldsymbol{y}_{t} \mid \boldsymbol{x}_{t}\right) p\left(\boldsymbol{x}_{t} \mid \boldsymbol{y}_{1: t-1}\right) d \boldsymbol{x}_{t}}
\end{aligned}
$$

In the prediction step (Equation 6), the posterior probability density function (pdf) $p\left(\boldsymbol{x}_{t} \mid \boldsymbol{y}_{1: t-1}\right)$ is obtained based on the fact that the transition pdf $p\left(x_{t} \mid \boldsymbol{x}_{t-1}\right)$ and the prior pdf at time step $t-1$ are known, whereas in the correction step (Eq. 7), the prior pdf is corrected using the information from the likelihood pdf $p\left(\boldsymbol{y}_{t} \mid \boldsymbol{x}_{t}\right)$ and the posterior pdf $p\left(\boldsymbol{x}_{t} \mid \boldsymbol{y}_{1 ; t}\right)$ is derived. The analytical solution of Eqs. (6) and (7) is difficult to determine since the evaluation of the integrals might be intractable. Particle filters are a set of algorithms which approximate the posterior pdf by a group of random samples. In more detail, the integrals are mapped to discrete sums:

$p\left(\boldsymbol{x}_{t} \mid \boldsymbol{y}_{1: t}\right) \approx \hat{p}\left(\boldsymbol{x}_{t} \mid \boldsymbol{y}_{1: t}\right)=\frac{1}{N} \sum_{i=1}^{N} \delta\left(\boldsymbol{x}_{t}-\boldsymbol{x}_{t, i}\right)$

where the particles $\left\{\boldsymbol{x}_{t, i} ; i=1 \ldots N\right\}$ should be sampled from the posterior pdf and $\delta$ is the Dirac measure. The Dirac measure is given by:

$\delta_{x}(X)= \begin{cases}0 & \text { if } x \notin X, \\ 1 & \text { if } x \in X .\end{cases}$

where $x$ is a possible element of set $X$.

At this point, drawing particles is unfeasible since the posterior pdf is unknown. Nevertheless, it is viable to draw particles from a known proposal pdf (also called importance pdf). This is the basis of the importance sampling principle. Sequential Importance Sampling (SIS) is the recursive version of the importance sampling MC method and the particle filters are based on the SIS approach. This approach 
approximates the posterior pdf by a set of weighted particles as follows:

$\hat{p}\left(\boldsymbol{x}_{t} \mid \boldsymbol{y}_{1: t}\right)=\sum_{i=1}^{N} \tilde{\mathbf{w}}_{t, i} \delta\left(\boldsymbol{x}_{t}-\boldsymbol{x}_{t, i}\right)$

where $\tilde{\mathbf{w}}_{t, i}$ are the normalized importance weights associated to the particles which are drawn from the proposal pdf. Considering that the system state evolves according to a Markov process, and applying recursion to the filtering problem, the recursive expression for the importance weights is given by:

$\mathbf{w}_{t, i}=\mathbf{w}_{t-1, i} \cdot \frac{p\left(\boldsymbol{y}_{t} \mid \boldsymbol{x}_{t, i}\right) p\left(\boldsymbol{x}_{t, i} \mid \boldsymbol{x}_{t-1, i}\right)}{q\left(\boldsymbol{x}_{t, i} \mid \boldsymbol{x}_{0: t-1, i}, \boldsymbol{y}_{1: t}\right)}$

The selection of the proposal pdf $q\left(., \boldsymbol{y}_{1: t}\right)$ is extremely important in the design stage of the SIS filter. The filter performance mainly depends on how well the proposal pdf approximates the posterior pdf. In Doucet et al. (2001), an optimal choice for the importance density function is proposed:

$q\left(\boldsymbol{x}_{t} \mid \boldsymbol{x}_{0: t-1}, \boldsymbol{y}_{1: t}\right)=p\left(\boldsymbol{x}_{t} \mid \boldsymbol{x}_{0: t-1}, \boldsymbol{y}_{1: t}\right)$

This pdf is optimal in the sense that it minimizes the variance of the importance weights, however, the application of Eq. (12) is complex from the implementation point of view. A common choice of the proposal is the transition prior function (Gordon et al., 1993; Kitagawa, 1996):

$q\left(\boldsymbol{x}_{t, i} \mid \boldsymbol{x}_{0: t-1, i}, \boldsymbol{y}_{1: t}\right)=p\left(\boldsymbol{x}_{t, i} \mid \boldsymbol{x}_{t-1, i}\right)$

A drawback of this approach is the lack of information regarding the model errors in the computation of the importance weights. This limitation can affect the performance of the particle filter. The choice of the transition prior as the proposal simplifies Equation 11 resulting in an expression where the importance weights depend on their past values and also on the likelihood pdf. In this application, the likelihood pdf is considered to be Gaussian. Thus, the particles are weighted according to:

$p\left(\boldsymbol{y}_{t} \mid \hat{\boldsymbol{x}}_{t, i}^{-}\right)=\frac{\exp \left(-\frac{1}{2}\left(\boldsymbol{y}_{t}-\mathbf{H}_{t} \hat{\boldsymbol{x}}_{t, i}^{-}\right)^{\mathbf{T}} \mathbf{R}^{-1}\left(\boldsymbol{y}_{t}-\mathbf{H}_{t} \hat{\boldsymbol{x}}_{t, i}^{-}\right)\right)}{(2 \pi)^{m / 2}|\mathbf{R}|^{1 / 2}}$

where $\mathbf{R}$ is the measurement error covariance matrix, which is set to $0.0005 \mathbf{I}$, with $\mathbf{I}$ the identity matrix and the uncertainty of $0.022 \mathrm{~cm}^{3} \mathrm{~cm}^{-3}(\sqrt{0.0005})$ is less than the valid upper limit of $0.05 \mathrm{~cm}^{3} \mathrm{~cm}^{-3}$ reported in Walker and Houser (2004). $|\mathbf{R}|$ is the determinant of matrix $\mathbf{R}$ and $m$ is the dimension of vector $\boldsymbol{y}_{t}$. The normalized weights are given by:

$\tilde{\mathbf{w}}_{t, i}=\frac{\mathbf{w}_{t, i}}{\sum_{i=1}^{N} \mathbf{w}_{t, i}}$

Finally, the best estimate of the state consists of the weighted mean of the particle set $\left\{\hat{\boldsymbol{x}}_{t, i}^{-}, \tilde{\mathbf{w}}_{t, i}\right\}$. The SIS filter poses the problem of particle depletion, this problem is caused by the increase of the variance over time as stated in Kong et al. (1994) and Doucet et al. (2001).
The plots in the upper part of Fig. 2 show the importance weight transition from a uniform distribution at $t=0$ to a normal distribution according to the Gaussian likelihood pdf at $t=1$. While in the plots located in the lower part, it is clearly noticeable that after a few model time steps, only one of the normalized importance weights reaches the value of 1 , and the remaining set of weights are reduced to negligible values. Consequently, a large number of samples are removed from the sample space, because their weights become numerically insignificant, generating a wrong approximation of the posterior pdf.

A heuristic approach to mitigate the degeneracy problem by increasing the particle set is impractical in most cases. The approach adopted in this work is the Sequential Importance Sampling with Resampling filter.

\subsubsection{Sequential Importance Sampling with Resampling (SISR)}

Resampling is basically the selection and replication of those particles with high importance weights. This additional step to the SIS filter involves mapping the Dirac random measure $\left\{\boldsymbol{x}_{t, i}, \tilde{\mathbf{w}}_{t, i}\right\}$ into an equally weighted random measure $\left\{\boldsymbol{x}_{t, i}, N^{-1}\right\}$. Gordon et al. (1993) proposed a methodology which consists of drawing samples uniformly from the discrete set $\left\{\boldsymbol{x}_{t, i}, \tilde{\mathbf{w}}_{t, i}\right\}$ and it is referenced as the Sampling Importance Resampling method (SIR). Beside the SIR method, more efficient selection techniques in terms of a reduction of the resampled particles variance have been developed such as the stratified resampling, systematic resampling and residual resampling (Arulampalam et al., 2002). Weerts and El Serafy (2006) found that the use of a minimum variance sampling technique such as the residual resampling improves the overall performance of the particle filter unlike the use of the SIR.

The SIR algorithm consists in the construction of the cumulative distribution of the particles set and the projection of a uniformly drawn sampling index $i$ onto the domain of the distribution. As a result of the projection, the resampling index $j$ is obtained and the particle set $\left\{\hat{\boldsymbol{x}}_{t, i}^{-}\right\}$is resampled according to this index, the resulting particle set $\left\{\hat{\boldsymbol{x}}_{t, j}^{+}\right\}$contains replications of those particles with high importance weight. An improved version of the SIR method is the stratified resampling approach introduced in Kitagawa (1996). This approach differs from the SIR only in the way how the sampling index $i$ is generated allowing for a reduction of the variance of the particles. The specific choice of the selection method among the stratified, systematic or residual resampling should not significantly affect the performance of the SISR filter since all three are designed to minimize the particle variance. The SISR particle filter applied in this study is the SIS filter with stratified resampling. 
The SIS with stratified resampling algorithm used is summarized as follows:

- FOR $t=1,2, \ldots$

- Propagate the particles in time. Draw $\hat{\boldsymbol{x}}_{t, i}^{-} \sim p\left(\hat{\boldsymbol{x}}_{t, i} \mid \hat{\boldsymbol{x}}_{t-1, i}^{+}\right)$

- IF ( $t$ corresponds to a DA time step)

- Importance sampling step

- For $i=1: N$

Compute $\mathbf{w}_{t, i}=p\left(\boldsymbol{y}_{t} \mid \hat{\boldsymbol{x}}_{t, i}^{-}\right)$

Normalize $\tilde{\mathbf{w}}_{t, i}=\frac{\mathbf{w}_{t, i}}{\sum_{i=1}^{N} \mathbf{w}_{t, i}}$

- Resampling step

- For $i=1: N$

Obtain the resampling index $\boldsymbol{j}$ vector.

Resample $\left\{\hat{\boldsymbol{x}}_{t, i}^{-}\right\} \Rightarrow\left\{\hat{\boldsymbol{x}}_{t, j}^{+}\right\}$

Assign $\tilde{\mathbf{w}}_{t, i}=\frac{1}{N}$

- END IF

\section{- END FOR}

The replication of the particles during the resampling step poses a problem when the set of resampled particles collapses in the worst case to a single particle due to a wrong selection of the importance pdf or due to a narrow likelihood pdf. Figure 3 shows the resampling index $\boldsymbol{j}$, which indicates the location of the particles to be resampled, at $4 \mathrm{DA}$ events. Subfigures (a) and (b) indicate a proper performance of the resampling algorithm where the particle replication is not extreme. On the other hand, the resampling index $\boldsymbol{j}$, as a result of the application of a hypothetical and too narrow likelihood pdf, is presented in subfigures (c) and (d), subfigure (c) indicates that the sample collapses to the particle values located at positions $27 \mathrm{th}$, 53th, and 60th. The extreme replication problem is noticeable mostly in subfigure (d) where almost all the particles collapse to the value of the particle located at the 54th position.

Andrieu et al. (1999), suggested for nonlinear systems the use of Markov chain Monte Carlo (MCMC) steps to deal with the particle degeneracy problem. However, the use of MCMC steps increases the computational time considerably due to need of new proposed particles which are sampled from the prior density function. The scope of this paper is limited to the application of the SISR filter assuming proper importance and likelihood density functions.

\subsubsection{SISR filter with parameter resampling (SISR-PR)}

In the PF, the uncertainty in the model is represented through samples referred to as particles. These samples are drawn from the importance density function (equal to the prior density function for the standard particle filter).
The uncertainty in the model is caused by uncertainty in the meteorological forcings, initial conditions and parameters. Thus, the generation of ensembles, presented in the experiment setup section, is fundamental since the ensemble should represent this model uncertainty. The perturbation of the parameters plays an important role in the generation of the ensemble due to the contribution of the parameters to the modelling errors.

The state estimation method aims at finding the optimal state value based on the information from the measurements. The estimated state value can positively or negatively affect the behaviour of other variables in the model. In this study, soil moisture is the state variable that will affect the baseflow.

Moradkhani et al. (2005b) presented the SISR-PR as a potential methodology to assess the uncertainty in the states and parameters of a hydrologic model. In this study we adopted the same methodology aiming at the correction of model flows after the assimilation of the states. The hydrologic literature on Data Assimilation with the Particle Filter focuses either on direct assimilation of discharge or on an evaluation of the improvement in the assimilated variable itself. In this paper, we assess whether the resampling of the parameters along with the states improves the behaviour of the model flows due to a proper combination between states and parameters.

The operation of the parameter resampling step is the following: after the resampling of the states, the same vector/matrix containing the particle indices to be resampled is used to resample the parameter set. The last action leads to a selection (replication or suppression) of parameters that are tied to a particular state realization.

An extreme replication of the parameter values poses the same problem as in the case of the state replication. Moreover, the ensemble will fail in the representation of the model uncertainty since the spread of the ensemble is decreased after the parameter resampling. In order to overcome this problem, the resampled parameter values are perturbed with the addition of white Guassian noise (Moradkhani et al., 2005b) and the variance (var) of the noise is set to a fraction of the optimal parameter value.

The SISR-PR filter applied in this study is summarized in the following algorithm:

- FOR $t=1,2, \ldots$

- Propagate the particles in time.

- IF ( $t$ corresponds to a DA time step)

- Importance sampling step

- Resampling step

- For $i=1: N$

Obtain the resampling index $\boldsymbol{j}$ vector.

Resample $\left\{\hat{\boldsymbol{x}}_{t, i}^{-}\right\} \Rightarrow\left\{\hat{\boldsymbol{x}}_{t, j}^{+}\right\}$

Resample the parameter set $\theta:\left\{\theta_{i}\right\} \Rightarrow\left\{\theta_{j}\right\}$

Perturb the resampled parameter set $\theta_{j}+$ 

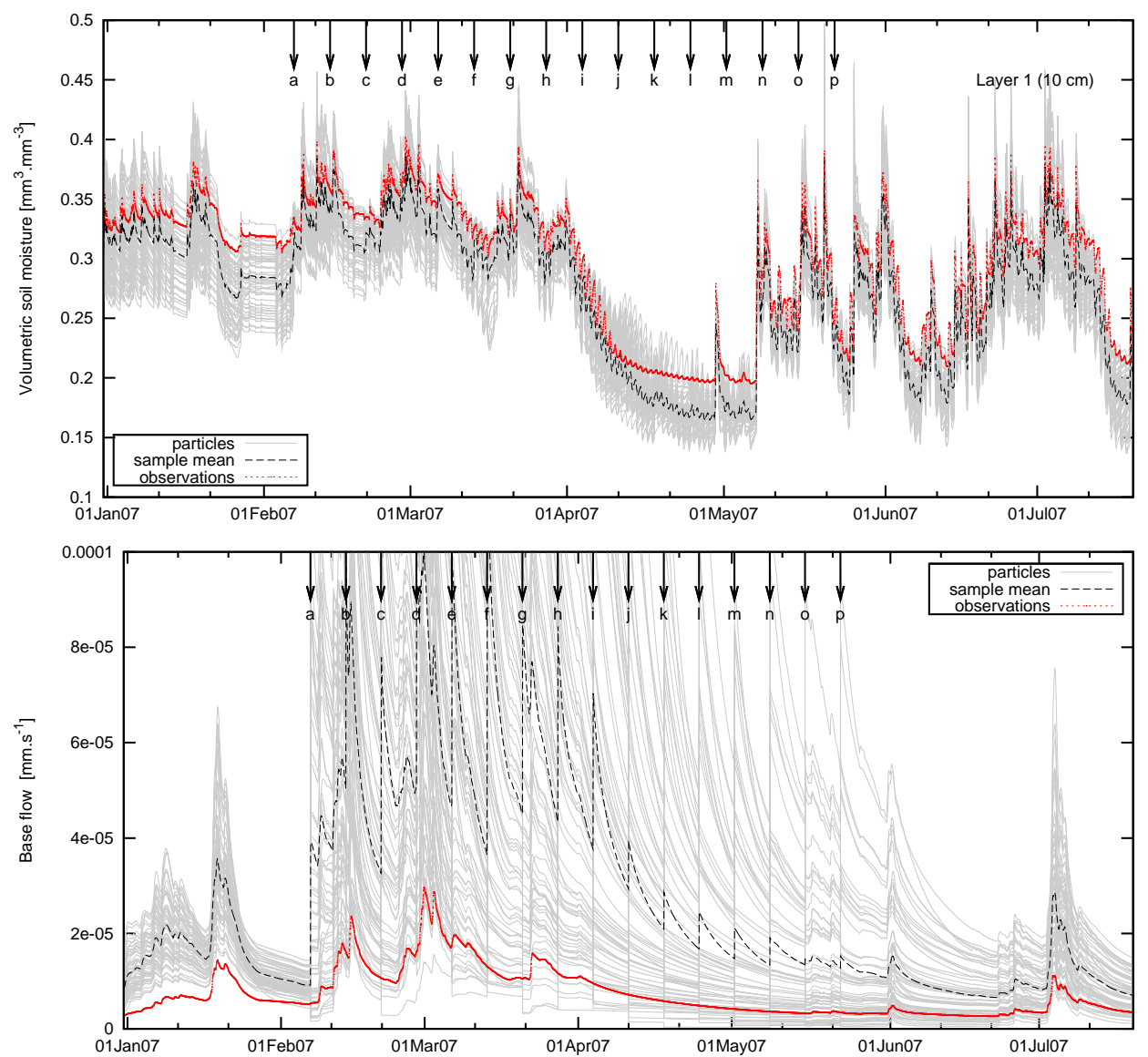

Fig. 5. SISR filter performance and assimilation impact on the baseflow. The soil moisture and baseflow time series correspond to the DA study performed with set 2 as the initial parameter set.

$$
\begin{aligned}
& N(0, \operatorname{var}) \\
& \text { Assign } \tilde{\mathbf{w}}_{t, i}=\frac{1}{N}
\end{aligned}
$$

- END IF

\section{- END FOR}

\section{Results and discussion}

The data assimilation experiments are validated by comparing soil moisture and baseflow assimilation results against synthetic observed soil moisture and baseflow values. The reference model integration without data assimilation is performed with parameter set 2 , while the assimilation integrations are performed with the different parameter sets 1,2 and 3.

Data assimilation is performed every week, with the first DA event at 8 February 2007 and the last at 24 May 2007. Every DA event is indicated by a black arrow in the figures and the simulation period corresponds to the first half of year 2007 (1 January-1 July). The Root Mean Square Error (RMSE), between the synthetic observed and modeled soil moisture and baseflow, is computed over the time period starting 1 day before the first DA event and 1 day after the last DA event (i.e., from 7 February to 25 May 2007).

\subsection{The SISR filter}

Figure 5 shows the performance of the SISR filter for soil moisture assimilation and the corresponding impact of the assimilation on the baseflow. According to the RMSE values: $3.65 \mathrm{vol} \%$ without assimilation and $2.19 \mathrm{vol} \%$ after assimilation, the improvement obtained from the SISR filter application is significant. However, when looking at the assimilation impact on the baseflow (lower part of figure 5) a different performance is observed. The filter performs negatively according to the RMSE value $\left(3.61 \times 10^{-5} \mathrm{~mm} \mathrm{~s}^{-1}\right.$ for the SISR filter) when compared to the model run without assimilation $\left(6.56 \times 10^{-6} \mathrm{~mm} \mathrm{~s}^{-1}\right)$.

The behaviour of the baseflow during the assimilation of soil moisture is the result of an inconsistent combination between resampled states and perturbed parameters mainly in the bottom soil layers which contribute to the generation of the baseflow. The replication of those state particles with 
higher weight in combination with the parameter values affect the baseflow behaviour negatively. More specifically, the rearrangement of the soil moisture particles in combination with parameter values for wet or dry moisture conditions generates huge baseflow peaks as can be seen in the lower part of Fig. 5.

In order to assign to each resampled state particle a consistent parameter value, the application of the parameter resampling is important and it is evaluated as an alternative to improve the filter performance and to have a positive impact on the baseflow.

\subsection{The SISR filter with parameter resampling}

The SISR filter with parameter resampling aims at a combination of estimated state values with consistent parameter values. This procedure should result in a positive impact on the land surface variables that dynamically depend (through the model, including the parameter configuration) on the assimilated soil moisture state variable. The parameters involved in the resampling step are listed in Table 1, parameters NwRb and NwRs are not considered for resampling.

Figure 6 shows the performance of the soil moisture assimilation and the impact of the assimilation on the baseflow for the SISR-PR filter without the perturbation of the resampled parameters. Looking at Figs. 4 and 6, the decrease in the dispersion of the soil moisture and baseflow particles is noticeable when the parameter resampling is performed. This reduction is indicated by the time-averaged ensemble spread <ensp $>$ (Eq. 1), calculated over the entire validation period with inclusion of the DA time steps, with values of $6.16 \times 10^{-4}$ and $5.92 \times 10^{-5}\left(\mathrm{~mm}^{3} \mathrm{~mm}^{-3}\right)^{2}$ for soil moisture and values of $1.34 \times 10^{-11}$ and $9.27 \times 10^{-12}\left(\mathrm{~mm} \mathrm{~s}^{-1}\right)^{2}$ for the baseflow.

Resampling the parameters along with the state SISR filter causes a reduction of the analysis error (the ensemble spread represents the uncertainty at the analysis step). An extreme reduction of the ensemble spread due to an extreme state and parameter particles replication needs to be avoided. Here, we propose the perturbation of the resampled parameters by using additive white Gaussian noise as the solution to the particles collapse problem. The predefined standard deviation of the noise is set to a fraction of the optimal parameter values, for the results presented in Fig. 7 the fraction is set to 0.01 of parameter set 2 . This fraction was obtained based on a proper representation of the baseflow ensemble through the calibration of the ensemble spread measure.

Figure 7 shows the SISR-PR filter performance with the perturbation of the resampled parameters. The upper part of this figure presents the performance for the soil moisture assimilation. The dynamics of the state ensemble is positively affected by the parameter resampling improving the overall performance of the filter and keeping the benefit of the state updating for a long time after the DA events. The benefit is quantified by the RMSE values correspond-
Table 2. RMSE $\left[\mathrm{mm}^{3} \mathrm{~mm}^{-3}\right.$ ] between the observed and simulated soil moisture for 3 parameter sets.

\begin{tabular}{llll}
\hline Filter & set 1 & set 2 & set 3 \\
\hline Ensemble & 2.89 & 3.65 & 3.38 \\
SISR filter & 2.33 & 2.19 & 1.61 \\
SISR-PR filter & 1.85 & 0.51 & 0.74 \\
\hline
\end{tabular}

Table 3. RMSE $\left[\mathrm{mm} \mathrm{s}^{-1}\right]$ between the observed and simulated baseflow for 3 parameter sets. SISR-PR is applied with parameter perturbation.

\begin{tabular}{llll}
\hline Filter & set 1 & set 2 & set 3 \\
\hline Ensemble & $1.39 \times 10^{-5}$ & $6.56 \times 10^{-6}$ & $5.89 \times 10^{-6}$ \\
SISR filter & $2.71 \times 10^{-5}$ & $3.61 \times 10^{-5}$ & $4.08 \times 10^{-5}$ \\
SISR-PR filter & $1.16 \times 10^{-5}$ & $3.40 \times 10^{-6}$ & $2.39 \times 10^{-6}$ \\
\hline
\end{tabular}

ing to $3.65 \mathrm{vol} \%$ without assimilation and 0.51 vol \% when the SISR-PR is applied. Moreover, the perturbation of the resampled parameters increases the ensemble spread from $5.91 \times 10^{-5}\left(\mathrm{~mm}^{3} \mathrm{~mm}^{-3}\right)^{2}$ to $7.97 \times 10^{-5}\left(\mathrm{~mm}^{3} \mathrm{~mm}^{-3}\right)^{2}$.

Additionally, the plot of the baseflow (see lower part of Fig. 7) shows graphically a considerable improvement on the behaviour when comparing to the assimilation effects of the SISR filter application. Figure 8 shows the impact of the assimilation on the bottom soil layers. The benefit of the parameter resampling in the top soil layer is propagated trough the bottom soil layers. As a consequence, the impact on the baseflow is positively affected. This improvement can be corroborated with the reduction in the RMSE values from $6.56 \times 10^{-6} \mathrm{~mm} \mathrm{~s}^{-1}$ when no assimilation is performed to $3.40 \times 10^{-6} \mathrm{~mm} \mathrm{~s}^{-1}$ when soil moisture DA is performed. The baseflow ensemble spread can be increased by the parameter perturbation. The ensemble spread values indicate an increase from $9.26 \times 10^{-12}\left(\mathrm{~mm} \mathrm{~s}^{-1}\right)^{2}$ to $1.51 \times 10^{-11}$ $\left(\mathrm{mm} \mathrm{s}^{-1}\right)^{2}$.

Figure 9 shows the evolution in time of the soil hydraulic model parameters for the top soil moisture layer. Parameters bsw $_{1}$, watsat 1 , hksat 1 converge to the "truth" (parameter values used in the generation of the synthetic observations) and parameter sucsat $_{1}$ converges to a different value. The correction of the soil moisture in the bottom layers and of the parameters during the first DA time steps allows for a correction of the baseflow even in the case when the baseflow measurements are not covered by the baseflow ensemble as can be seen in Figs. 6 and 7 .

An overall conclusion based on the good RMSE values obtained for soil moisture and baseflow is that the addition of the parameter resampling to the SIR filter is effective in removing the bias through an indirect calibration of the modeled particles. 

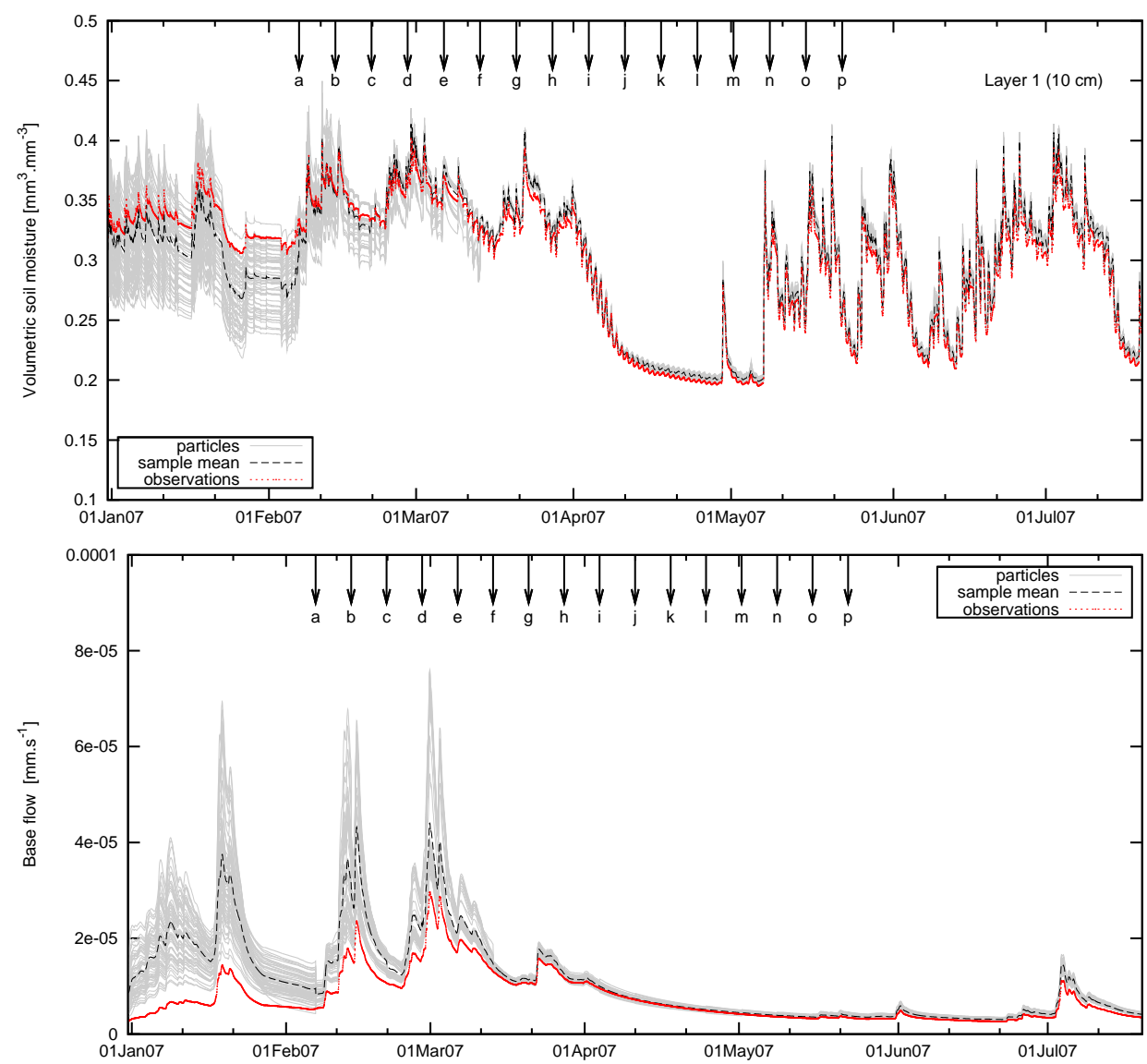

Fig. 6. SISR-PR filter performance and assimilation impact on the baseflow without parameter perturbation. The soil moisture and baseflow time series correspond to the DA study performed with set 2 as the initial parameter set.

Table 4. RMSE $\left[\mathrm{mm}^{3} \cdot \mathrm{mm}^{-3}\right]$ between the observed and simulated soil moisture for 3 DA frequencies.

\begin{tabular}{llll}
\hline Filter & DA every week & DA every 2 weeks & DA every 4 weeks \\
\hline Ensemble & 3.65 & 3.65 & 3.65 \\
SISR filter & 2.19 & 2.34 & 2.60 \\
SISR-PR filter & 0.51 & 0.76 & 1.22 \\
\hline
\end{tabular}

\subsection{Sensitivity study}

The performance of the SISR-filter and SISR-PR filter with parameter perturbation are further analyzed for 3 different initial parameter sets, each identified by the automatic calibration algorithm with a similar optimization index value. The filter performance is analyzed through the comparison of the RMSE values. The parameter sets 1,2 and 3 represent 3 different local minima in the parameter space, the idea behind this is to check the robustness of the parameter resampling algorithm.

Table 2 presents the RMSE values between the estimated and observed volumetric soil moisture at the surface for every filter and for every parameter set. Although the SISR-PR
RMSE values are different, due to different system dynamics the SISR-PR filter outperforms the rest of the filters indicating robustness of the algorithm. Additionally, according to Table 3 the positive impact on the baseflow persists among the three cases.

Considering the assimilation of remote sensed soil moisture data, the availability of data is of main importance in the application of the assimilation algorithm. Therefore, the SISR-PR performance is tested for $3 \mathrm{DA}$ frequencies. Additionally to the DA frequency corresponding to $16 \mathrm{DA}$ events, the methodology is evaluated for 8 DA events with 1 event every 2 weeks and 4 DA steps with 1 event every four weeks. 

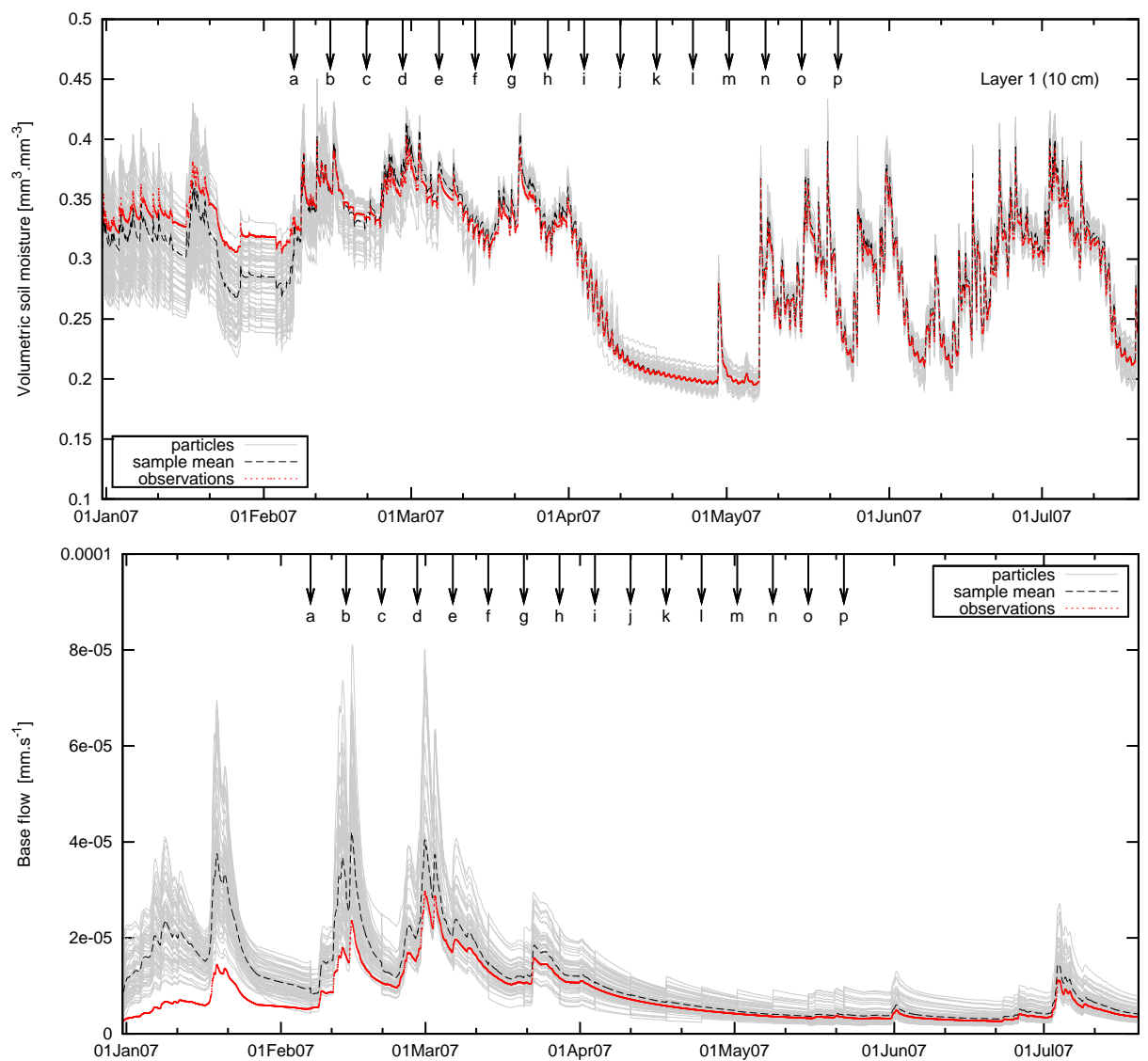

Fig. 7. SISR-PR filter performance and assimilation impact on the baseflow with parameter perturbation. The soil moisture and baseflow time series correspond to the DA study performed with set 2 as the initial parameter set.

Table 5. RMSE $\left[\mathrm{mm} \cdot \mathrm{s}^{-1}\right.$ ] between the observed and simulated baseflow for 3 DA frequencies. SISR-PR is applied with parameter perturbation.

\begin{tabular}{llll}
\hline Filter & DA every week & DA every 2 weeks & DA every 4 weeks \\
\hline Ensemble & $6.56 \times 10^{-6}$ & $6.56 \times 10^{-6}$ & $6.56 \times 10^{-6}$ \\
SISR filter & $3.61 \times 10^{-5}$ & $3.26 \times 10^{-5}$ & $2.51 \times 10^{-5}$ \\
SISR-PR filter & $3.40 \times 10^{-6}$ & $4.23 \times 10^{-6}$ & $4.37 \times 10^{-6}$ \\
\hline
\end{tabular}

Tables 4 and 5 show the RMSE values for the 3 DA frequencies for soil moisture and baseflow respectively. The values indicate a notorious improvement when using the SISR-PR and the positive impact on the baseflow is maintained for the $3 \mathrm{DA}$ frequencies. An additional sensitivity test is recommended concerning the impact of the noise level and the magnitude of the truth parameter set on the behaviour of the baseflow since different performances have been noted when using different parameter values. In Table 5, the RMSE values corresponding to the performance of the SISR filter for the 3 DA frequencies indicate the degeneracy of the baseflow due to an inconsistent combination of states and parameters.

\section{Summary and conclusions}

The SISR filter has been evaluated for the performance in assimilation of soil moisture and the impact thereof on baseflow fluxes. The filter performs relatively good for the correction of the modeled soil moisture, although it should be noted the presence of bias. The impact of the soil moisture assimilation on the baseflow results indicates a strong negative effect. The SISR-PR approach is presented as a solution to this shortcoming in the SISR filter performance.

The SISR-PR filter methodology strives on the correction of the consistency between parameters and soil moisture states replicating the consistent parameters and rejecting 

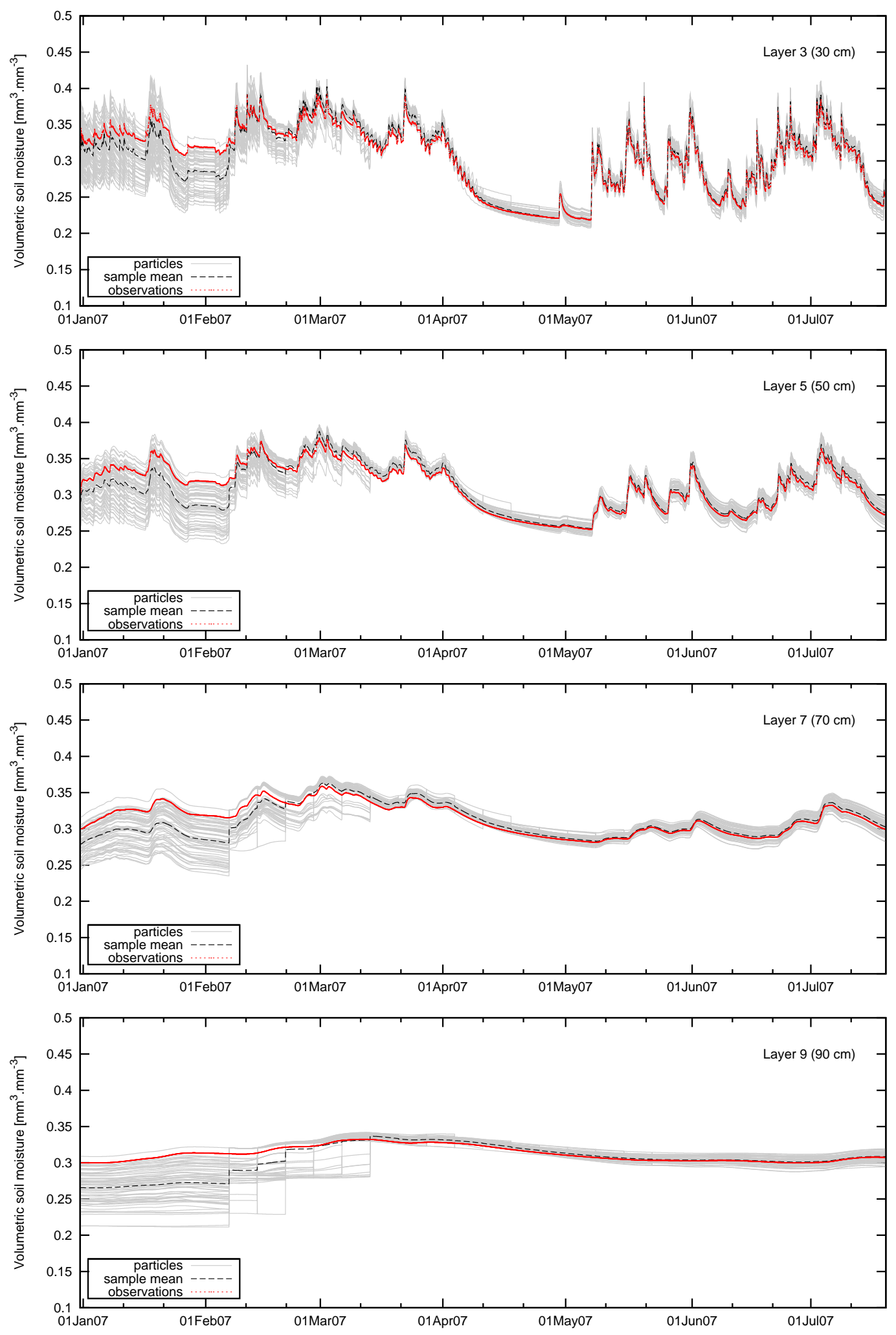

Fig. 8. Impact of the assimilation of soil moisture in the top layer on the bottom layers when the SISR-PR filter is applied with parameter perturbation. 

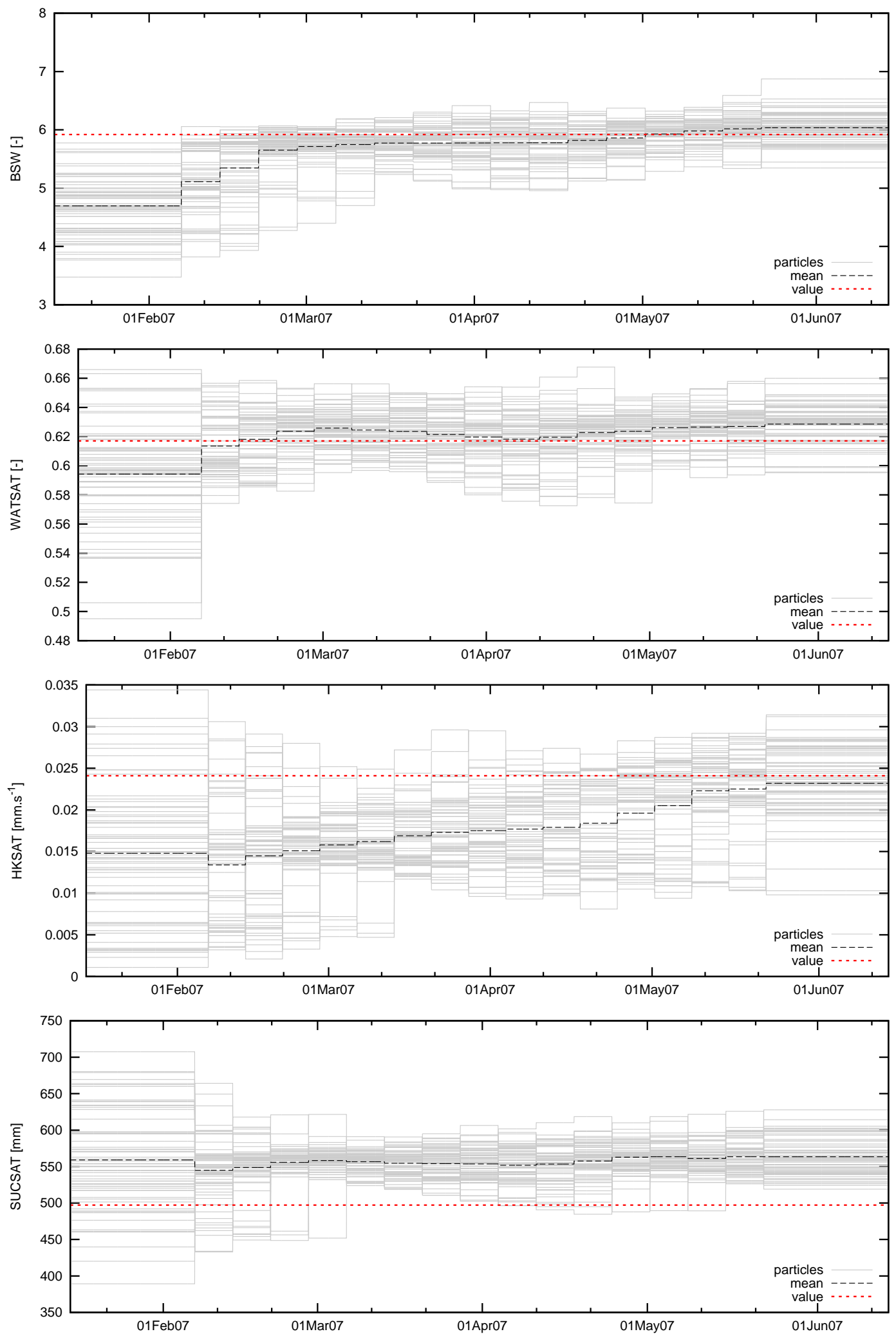

Fig. 9. Evolution in time of the soil parameters in the top layer when SISR-PR filter is applied with parameter perturbation. 
the erratic parameter values. Results indicate a notorious improvement of the performance not only in the estimation of the soil moisture but also in the influence on the baseflow.

Yet, a severe replication affects the parameter diversity and leads to an improper representation of the posterior pdf when assimilating data. The perturbation of the resampled parameter set by a white Gaussian noise with zero mean and predefined standard deviation mitigates the side-effects of the replication.

The robustness of the SISR-PR filter has been tested through the evaluation of the SISR-PR filter for different parameter sets and different assimilation frequencies. An overall conclusion is that the addition of parameter resampling is effective in removing the bias.

\section{Supplement related to this article is available online at: http://www.hydrol-earth-syst-sci.net/16/375/2012/ hess-16-375-2012-supplement.zip.}

Acknowledgements. The work in this paper has been funded by the Belgian Science Policy for the HYDRASENS project in the frame of the STEREO II programme. Gabriëlle De Lannoy is a postdoctoral researcher funded by the Foundation of Scientific Research of the Flemish Community (FWO-Vlaanderen). The authors are very grateful to the reviewers, in particular Carsten Montzka, Hamid Moradkhani, Karthik Nagarajan and the editor Albrecht Weerts for their valuable contribution to the development of this paper.

Edited by: A. Weerts

\section{References}

Andersson, L.: Improvement of runoff models, what way to go?, Nord. Hydrol., 23, 315-332, 1992.

Andrieu, C., de Freitas, J. F. G., and Doucet, A.: Sequential Bayesian Estimation And Model Selection Applied To Neural Networks, CUED-F-INFENG-TR-341, Cambridge University Engineering Department, March 1999.

Arulampalam, M. S., Maskell, S., and Gordon, N.: A tutorial on particle filters for online nonlinear/non-Gaussian Bayesian tracking, IEEE T. Signal Proc., 50, 174-188, 2002.

Brocca, L., Melone, F., Moramarco, T., Wagner, W., Naeimi, V., Bartalis, Z., and Hasenauer, S.: Improving runoff prediction through the assimilation of the ASCAT soil moisture product, Hydrol. Earth Syst. Sci., 14, 1881-1893, doi:10.5194/hess-141881-2010, 2010.

Caparrini, F., Castelli, F., and Entekhabi, D.: Variational estimation of soil and vegetation turbulent transfer and heat flux parameters from sequences of multisensor imagery, Water Resour. Res., 40, W12515, doi:10.1029/2004WR003358, 2004.

De Lannoy, G. J. M., Houser, P. R., Pauwels, V. R. N., and Verhoest, N. E. C.: Assessment of model uncertainty for soil moisture through ensemble verification, J. Geophys. Res., 111, D10101, doi:10.1029/2005JD006367, 2006a.
De Lannoy, G. J. M.: Assimilation of soil moisture observations into a spatially distributed hydrologic model, Ph.D. thesis, Ghent University, 2006b.

De Lannoy, G. J. M., Houser, P. R., Pauwels, V. R. N., and Verhoest, N. E. C.: State and bias estimation for soil moisture profiles by an ensemble Kalman filter: Effect of assimilation depth and frequency, Water Resour. Res., 43, W06401, doi:10.1029/2006WR005100, 2007a.

De Lannoy, G. J. M., Reichle, R. H., Houser, P. R., Pauwels, V. R. N., and Verhoest, N. E. C.: Correcting for forecast bias in soil moisture assimilation with the ensemble Kalman filter, Water Resour. Res., 43, W09410, doi:10.1029/2006WR005449, $2007 \mathrm{~b}$.

Doucet, A., Gordon, N., and Krishnamurthy, V.: Particle filters for state estimation of jump Markov linear systems, IEEE T. Signal Proces., 49, 613-624, doi:10.1109/78.905890, 2001.

Duan, Q. Y., Gupta, V. K., and Sorooshian, S.: Shuffled complex evolution approach for effective and efficient global minimization, J. Optim. Theory Appl., 76, 501-521, 1993.

Dunne, S. and Entekhabi, D.: An ensemble-based reanalysis approach to land data assimilation, Water Resour. Res., 41, W02013, doi:10.1029/2004WR003449, 2005.

Entekhabi, D., Nakamura, H., and Njoku, E. G.: Solving the inverse-problem for soil moisture and temperature profiles by sequential assimilation of multifrequency remotely sensed observations, IEEE T. Geosci. Remote., 32, 438-448, 1994.

Franssen, H. J. H. and Kinzelbach, W.: Real-time groundwater flow modeling with the Ensemble Kalman Filter: Joint estimation of states and parameters and the filter inbreeding problem, Water Resour. Res., 44, W09408, doi:10.1029/2007WR006505, 2008.

Giustarini, L., Matgen, P., Hostache, R., Montanari, M., Plaza, D., Pauwels, V. R. N., De Lannoy, G. J. M., De Keyser, R., Pfister, L., Hoffmann, L., and Savenije, H. H. G.: Assimilating SAR-derived water level data into a hydraulic model: a case study, Hydrol. Earth Syst. Sci., 8, 2103-2144, doi:10.5194/hessd-8-2103-2011, 2011.

Gordon, N., Salmond, D., and Smith, A.: Novel approach to nonlinear/non-Gaussian Bayesian state estimation, Radar and Signal Processing, IEEE Proc. F, 140, 107-113, 1993.

Heathman, G. C., Starks, P. J., Ahuja, L. R., and Jackson, T. J.: Assimilation of surface soil moisture to estimate profile soil water content, J. Hydrol., 279, 1-17, 2003.

Houser, P. R., Shuttleworth, W. J., Gupta, H. V., Famiglietti, J. S., Syed, K. H., and Goodrich, D. C.: Integration of Soil Moisture Remote Sensing and Hydrologic Modeling using Data Assimilation, Water Resour. Res., 34, 3405-3420, 1998.

Kalman, R. E.: A New Approach to Linear Filtering and Prediction Problems, Transactions of the ASME - J. Basic Eng., 82, 35-45, 1960.

Kitagawa, G.: Monte Carlo Filter and Smoother for Non-Gaussian Nonlinear State Space Models, J. Comput. Graph. Stat., 5, 1-25, 1996.

Kong, A., Liu, J. S., and Wong, W. H.: Sequential Imputations and Bayesian Missing Data Problems, J. Am. Stat. Assoc., 89, 278288, 1994.

Liu, J. S. and Chen, R.: Sequential Monte Carlo Methods for Dynamic Systems, J. Am. Stat. Assoc., 93, 1032-1044, 1998.

Matgen, P., Montanari, M., Hostache, R., Pfister, L., Hoffmann, L., Plaza, D., Pauwels, V. R. N., Lannoy, G. J. M. D., Keyser, R. D., and Savenije, H. H. G.: Towards the sequential assimilation of 
SAR-derived water stages into hydraulic models using the Particle Filter: proof of concept, Hydrol. Earth Syst. Sci., 14, 17731785, 2010, http://www.hydrol-earth-syst-sci.net/14/1773/2010/.

Montzka, C., Moradkhani, H., Weihermüller, L., Franssen, H.-J. H., Canty, M., and Vereecken, H.: Hydraulic parameter estimation by remotely-sensed top soil moisture observations with the particle filter, J. Hydrol., 399, 410-421, 2011.

Moradkhani, H., Sorooshian, S., Gupta, H. V., and Houser, P. R.: Dual state-parameter estimation of hydrological models using ensemble Kalman filter, Adv. Water Resour., 28, 135-147, doi:10.1016/j.advwatres.2004.09.002, 2005a.

Moradkhani, H., Hsu, K.-L., Gupta, H., and Sorooshian, S.: Uncertainty assessment of hydrologic model states and parameters: Sequential data assimilation using the particle filter, Water Resour. Res., 41, W05012, 2005 b.

Nagarajan, K., Judge, J., Graham, W. D., and Monsivais-Huertero, A.: Particle Filter-based assimilation algorithms for improved estimation of root-zone soil moisture under dynamic vegetation conditions, Adv. Water Resour., 34, 433-477, 2010.

Paniconi, C., Maroccu, M., Putti, M., and Verbunt, M.: Newtonian nudging for a Richards equation-based distributed hydrological mode, Adv. Water Resour., 26, 161-178, 2002.

Pauwels, V. R. N., Hoeben, R., Verhoest, N. E. C., and De Troch, F. P.: The importance of the spatial patterns of remotely sensed soil moisture in the improvement of discharge predictions for small-scale basins through data assimilation, J. Hydrol., 251, 88102, 2001

Pauwels, V. R. N., Hoeben, R., Verhoest, N. E. C., De Troch, F. P., and Troch, P. A.: Improvement of TOPLATS-based discharge predictions through assimilation of ERS-based remotely sensed soil moisture values, Hydrol. Process., 16, 995-1013, 2002.
Reichle, R. H., Walker, J. P., Koster, R. D., and Houser, P. R.: Extended versus Ensemble Kalman filtering for land data assimilation, J. Hydrometeorol., 3, 728-740, 2002.

Seuffert, G., Wilker, H., Viterbo, P., Drusch, M., and Mahfouf, J. F.: The usage of screen-level parameters and microwave brightness temperature for soil moisture analysis, J. Hydrometeorol., 5, 516-531, 2004.

Troch, P. A., Smith, J. A., Wood, E. F., and de Troch, F. P.: Hydrologic controls of large floods in a small basin: central Appalachian case study, J. Hydrol., 156, 285-309, doi:10.1016/0022-1694(94)90082-5, 1994.

Walker, J. P. and Houser, P. R.: Requirements of a global near-surface soil moisture satellite mission: accuracy, repeat time, and spatial resolution, Adv. Water Resour., 27, 785-801, doi:10.1016/j.advwatres.2004.05.006, 2004.

Walker, J. P., Willgoose, G. R., and Kalma, J. D.: Threedimensional soil moisture profile retrieval by assimilation of near-surface measurements: Simplified Kalman filter covariance forecasting and field application, Water Resour. Res., 38, 1301, doi:10.1029/2002WR001545, 2002.

Wang, D., Chen, Y., and Cai, X.: State and parameter estimation of hydrologic models using the constrained ensemble Kalman filter, Water Resour. Res., 45, W11416, doi:10.1029/2008WR007401, 2009.

Weerts, A. H. and El Serafy, G. Y. H.: Particle filtering and ensemble Kalman filtering for state updating with hydrological conceptual rainfall-runoff models, Water Resour. Res., 42, W09403, doi:10.1029/2005WR004093, 2006.

Zhang, J., Wang, W., and Leung, L.: Contribution of landatmosphere coupling to summer climate variability over the contiguous United States, J. Geophys. Res., 113, D22109, doi:10.1029/2008JD010136, 2008. 